Meta

Journal des traducteurs

Translators' Journal

\title{
Una mirada al pasado: aportes de la historia al estudio de la interpretación
}

\section{Marjorie Agrifoglio}

Volume 50, numéro 4, décembre 2005

Pour une traductologie proactive - Actes

For a Proactive Translatology - Proceedings

Por una traductología proactiva - Actas

URI : https://id.erudit.org/iderudit/019926ar

DOI : https://doi.org/10.7202/019926ar

Aller au sommaire du numéro

Éditeur(s)

Les Presses de l'Université de Montréal

ISSN

0026-0452 (imprimé)

1492-1421 (numérique)

Découvrir la revue

Citer cet article

Agrifoglio, M. (2005). Una mirada al pasado: aportes de la historia al estudio de la interpretación. Meta, 50(4). https://doi.org/10.7202/019926ar
Résumé de l'article

L'objet de cet article est de souligner l'importance de la recherche historique pour la théorie de l'interprétation, à partir de l'illustration de notre projet de thèse doctorale sur le rôle des interprètes dans les négociations des traités de cession de territoire conclus avec les Amérindiens canadiens au XIX ${ }^{\mathrm{e}}$ siècle. Le travail met l'accent, dans un premier temps, sur les liens entre l'histoire et la théorie en traductologie, ainsi que sur l'intérêt des recherches socioculturelles en interprétation. Ainsi, nous postulons que l'histoire peut ouvrir la voie à un " cultural turn » en interprétation. Ensuite, nous examinons le contexte des négociations des traités canadiens et nous mentionnons le nom de quelques interprètes qui y ont participé. Ceux-ci se divisent clairement en deux groupes : les Métis et les missionnaires catholiques et protestants. Enfin, nous proposons deux hypothèses sur le rôle actif qu'ont joué ces interprètes dans le but de contribuer à une meilleure compréhension de la dimension socioculturelle de l'interprétation, surtout lorsque les deux parties impliquées ont de grandes différences culturelles et sociales, comme en interprétation communautaire.
Ce document est protégé par la loi sur le droit d'auteur. L'utilisation des services d’Érudit (y compris la reproduction) est assujettie à sa politique d'utilisation que vous pouvez consulter en ligne.

https://apropos.erudit.org/fr/usagers/politique-dutilisation/ 


\title{
Una mirada al pasado: aportes de la historia al estudio de la interpretación
}

\author{
MARJORIE AGRIFOGLIO \\ Université de Montréal, Montréal, Canada \\ agrifogliom@videotron.ca
}

\begin{abstract}
RÉSUMÉ
L'objet de cet article est de souligner l'importance de la recherche historique pour la théorie de l'interprétation, à partir de l'illustration de notre projet de thèse doctorale sur le rôle des interprètes dans les négociations des traités de cession de territoire conclus avec les Amérindiens canadiens au $\mathrm{XIX}^{\mathrm{e}}$ siècle. Le travail met l'accent, dans un premier temps, sur les liens entre l'histoire et la théorie en traductologie, ainsi que sur l'intérêt des recherches socioculturelles en interprétation. Ainsi, nous postulons que l'histoire peut ouvrir la voie à un «cultural turn » en interprétation. Ensuite, nous examinons le contexte des négociations des traités canadiens et nous mentionnons le nom de quelques interprètes qui y ont participé. Ceux-ci se divisent clairement en deux groupes : les Métis et les missionnaires catholiques et protestants. Enfin, nous proposons deux hypothèses sur le rôle actif qu'ont joué ces interprètes dans le but de contribuer à une meilleure compréhension de la dimension socioculturelle de l'interprétation, surtout lorsque les deux parties impliquées ont de grandes différences culturelles et sociales, comme en interprétation communautaire.
\end{abstract}

\begin{abstract}
The purpose of this paper is to underline the importance of historical research for interpreting theory by presenting our $\mathrm{PhD}$ research on the role of interpreters during the negotiation of Canadian Indian treaties in the $19^{\text {th }}$ century. Firstly, the relation between history and theory in translation studies is emphasized, together with the need for a socio-cultural approach in interpreting studies. History is viewed as the first stage towards a "cultural turn" in interpreting. Secondly, the context of Canadian Indian treaties' negotiations is examined, and some details about the interpreters are provided. Interpreters belong to two groups: the Métis and the missionaries, both Catholic and Protestant. To conclude, we propose two hypotheses on the active role that interpreters played during these negotiations. This study aims to contribute to a better understanding of the socio-cultural dimension of interpreting, especially when great cultural and social differences separate both parties, such as in community interpreting.
\end{abstract}

MOTS-CLÉS/KEYWORDS

interpreting, history, Indians, treaties, Canada

\section{Introducción}

Una mirada al pasado. ¿Qué puede decir el pasado sobre la interpretación? Puede decir, por ejemplo, que el 18 de agosto de 1876, en Fort Carlton, hoy Saskatchewan (Canadá), comenzaron las negociaciones para la firma del Tratado No. 6 entre las autoridades canadienses, encabezadas por el gobernador Morris, y los indios crees de las praderas, representados por el jefe Mista-wa-sis. Formaban parte de la delegación gubernamental dos intérpretes, el Reverendo John McKay y Peter Ballenden. Los indios habían llevado también a su propio intérprete, Peter Erasmus, cuyas memorias constituyen uno de los pocos testimonios que dejaron los protagonistas de este hecho histórico. Erasmus menciona un incidente que enturbió la interpretación en Fort Carlton:

Governor Morris started his address with Rev. McKay interpreting. (...) McKay interpretations were mixed with Swampy words. Mista-wa-sis jumped to his feet and said: "We are not Swampy Crees. We are Plain 
Crees and demand to be spoken in our language.” McKay understood, was confused and sat down. (...)

Ballenden was called up. (...) He made an excellent interpretation of the Governor's words but in a voice so low that it could not be heard beyond the first ten rows of men seated on the ground. The men in the back rows got to their feet and demanded that he speak in a louder voice (...). The Governor said: "All right, Erasmus. Let this be your chance to justify your chief's confidence in your work.” (Erasmus 1999: 242-243).

Si bien raya en lo anecdótico, este testimonio confirma que, para poder hacer un buen trabajo, el intérprete debe conocer muy bien las dos lenguas y poseer habilidades oratorias, dos de los principales requisitos que menciona hoy en día cualquier manual de interpretación. Al examinar la actuación de los intérpretes en el pasado, el intérprete contemporáneo puede corroborar los preceptos que recibió en su formación y entender la evolución de la profesión, entre otras cosas. Como se intentará demostrar a continuación, las "miradas al pasado" revisten un gran interés para la interpretación y la traductología, tanto desde el punto de vista práctico como teórico.

El objetivo de este trabajo es resaltar el aporte de la investigación histórica para el estudio de la interpretación a partir de nuestro proyecto de tesis doctoral sobre la historia de la interpretación en Canadá. En primer lugar, se explicará por qué es pertinente indagar sobre el pasado de la interpretación en traductología; en segundo lugar, se presentará el proyecto de tesis y, como conclusión, se hará hincapié en algunos aspectos que pueden servir de base para desarrollar propuestas teóricas sobre el papel del intérprete.

\section{Importancia de la investigación histórica en interpretación}

Como señala Baigorri (2003), la historia forma parte del progreso de toda disciplina, pues contribuye a rescatar la memoria colectiva. Baigorri ha planteado también en sus trabajos (2003, 2004b) que una historia escrita con rigor puede predecir hasta cierto punto el futuro de la profesión. Por ejemplo, el rechazo que manifestaron los primeros intérpretes de la posguerra por la simultánea se asemeja al temor que muchos intérpretes sienten hoy en día por la interpretación simultánea a distancia.

Además del valor informativo y formativo de la historia, desde el punto de vista teórico, el estudio de la interpretación en el pasado puede ser la primera etapa del camino que lleva a la formulación de teorías. Según Delisle (2003), la teoría y la historia están estrechamente relacionadas y el historiador de la traducción procede a la "génesis del pensamiento teórico". Este vínculo ya ha dado sus frutos en la teoría de la traducción, con trabajos como los de Hermans, Venuti, Pym, la "Escuela de la Manipulación" y los estudios post-coloniales y feministas, que estuvieron precedidos por una labor histórica. Dichos enfoques tuvieron el mérito de rebasar los límites de las perspectivas lingüísticas y comunicativas poniendo de manifiesto la influencia de factores políticos, sociales y culturales en la actividad traductora.

En interpretación, si bien se han realizado estudios históricos muy valiosos en la última década (Baigorri 2004a y 2004b, Cáceres Würsig 2004, Castilho País 2003, Demers 2003, Gaiba 1998, entre otros), la historia sigue ocupando un lugar marginal en la investigación. Conviene, por lo tanto, destacar su importancia y justificar su necesidad.

Al igual que en traducción, los trabajos históricos pueden enriquecer y ampliar la visión que se tiene de la interpretación, como explica Delisle (2003: 7):

Quand il tient compte de la dimension historique de la traduction, le théoricien est obligé (...) d'appliquer à sa réflexion la formule sociolinguistique bien connue : qui traduit quoi, pour qui, quand, où, pourquoi et dans quelles circonstances.

La historia de la traducción ha demostrado que no siempre se ha traducido de la misma manera, por lo que cabe pensar que tampoco se ha interpretado de una sola forma. La distancia temporal entre el objeto de estudio y el investigador permite cuestionar y evaluar los postulados teóricos. Una investigación que examine la actuación del intérprete en su contexto histórico, enfocándose "en el hombre" (Pym 1998), y que tome en cuenta el grupo étnico, la clase social y las motivaciones del intérprete, puede precisar y redefinir conceptos como la neutralidad, la ética, la 
visibilidad y el poder de los mediadores. En otras palabras, la historia puede generar un "cultural turn" en interpretación, como propugna Cronin (2002):

I want to argue here for the development of a cultural turn in interpreting studies. (...) There is a need for a material history of interpreting that would examine all forms of interpreting as they are grounded in the economic, political and cultural conditions of people's lives (...). Such an approach would not only greatly broaden the scope for research on contemporary situations but would also provide an impetus for historical work in interpreting to move from the descriptive to the analytical. (Cronin 2002: 391).

A continuación se ilustrará cómo un trabajo histórico puede apuntalar la dimensión sociocultural de la teoría de la interpretación.

\section{Los intérpretes en las negociaciones de los tratados de cesión de territorio con los amerindios canadienses $(\mathbf{1 8 5 0 - 1 9 2 3})$}

\subsection{Presentación y objetivos del estudio}

Desde su llegada a Canadá, tanto los franceses como los ingleses se vieron obligados a negociar con los amerindios que poblaban los nuevos territorios. Al principio, la motivación de los europeos era comercial y militar, por lo que realizaban alianzas de paz, comercio y amistad con los distintos grupos indígenas. Al comienzo del siglo XIX, cuando ya Gran Bretaña se había apoderado de los territorios colonizados por los franceses en Canadá, las relaciones con los amerindios tomaron un matiz diferente. Los colonizadores necesitaban más tierras para proseguir su expansión hacia el Oeste. Según la legislación inglesa, los dueños de los territorios vírgenes eran sus pobladores ancestrales (los amerindios). Para poder colonizar las tierras, las autoridades inglesas debían obtener la cesión de este derecho de propiedad. Fue entonces cuando empezaron a firmar tratados en los que los amerindios renunciaban a todos sus derechos territoriales. Estos tratados siguen en vigor en Canadá y muchos se han revisado a raíz de las protestas de varios grupos indígenas, quienes argumentan que sus antepasados firmaron los tratados sin entender realmente todos los términos y basándose en las promesas orales que se realizaron en las negociaciones y que nunca se pusieron por escrito (Morris 1880, Fumoleau 1973, Grammond 1995). En 1850, la firma de los tratados Robinson dio inicio al proceso de firma de los principales tratados de cesión de territorio en Canadá, los cuales se caracterizan por la gran superficie de las áreas cedidas y la confinación de los amerindios en las "reservas". Entre 1850 y 1867, la Corona británica firmó tres tratados con los amerindios: los dos tratados Robinson y el tratado de la isla Manitoulin. Después de la Confederación canadiense, entre 1871 y 1923, el gobierno prosiguió la política de los tratados y firmó los 11 tratados numerados y los tratados Williams. Estos tratados cubren parte de la provincia de Ontario, casi todo el territorio de las provincias de las Praderas y parte de los Territorios del Noroeste y de Colombia Británica. El estudio propuesto se limitará a los tratados sobre cesión de territorio firmados en Canadá entre 1850 y 1923 (15 tratados).

En la célebre obra Los traductores en la historia, de Delisle y Woodsworth (1995), a los intérpretes se les califica de "testigos privilegiados de la historia". Sin embargo, fuera del ámbito de la historia de la traducción, por lo general se disimula la participación de los mediadores. Son pocos los historiadores y cronistas que mencionan el nombre de los intérpretes y dan detalles sobre ellos. Este enmascaramiento de los intérpretes no es un fenómeno reciente, pues se observa incluso en las fuentes antiguas que dan información de primera mano. Como ya han señalado varios teóricos (Cronin 2002, Alonso Araguás 2003), en los relatos de la conquista y los viajes al Nuevo Mundo, una vez que se establece el contacto inicial, los exploradores europeos y los indígenas parecen comunicarse como por arte de magia, cuando es obvio que tenía que haber un intérprete entre ellos.

Ahora bien, en la historia de las relaciones entre los amerindios canadienses y los europeos, las negociaciones de los tratados de cesión de territorio, en especial las de los tratados numerados de 1871 a 1921, constituyen un caso particular. Se sabe que en estas negociaciones intervinieron intérpretes, no sólo debido a la barrera lingüística entre las dos partes, sino sobre todo porque su 
nombre y firma aparecen en los tratados, en la frase: "Signed in the date above written after having been read and interpreted to the Indians by...". En otras palabras, en este caso se resalta la participación del intérprete. Si bien puede parecer una mera formalidad, cabe preguntarse qué papel desempeñaron los intérpretes en esa situación.

El objetivo general de la investigación que estamos realizando es analizar el papel de los intérpretes en las negociaciones de los tratados de cesión de territorio con los amerindios canadienses. Específicamente se trata de saber quiénes fueron esos intérpretes, por qué y por quién fueron seleccionados, en qué condiciones trabajaban, qué formación o función tenían, y cómo los veían los distintos participantes.

\subsection{Los intérpretes}

A partir de los tratados y de algunas fuentes primarias y secundarias, hasta ahora han sido identificados veintitrés intérpretes. Estos intérpretes participaron en las negociaciones de los tratados numerados 1 a 7, firmados entre 1871 y 1877 en todo el territorio fértil de las praderas canadienses. El principal rasgo en común entre los mediadores es que la interpretación no era su principal actividad, lo cual no es sorprendente, pues la profesionalización se produjo en el siglo XX. Se distinguen dos grupos principales:

- Los "Métis" (mestizos): Como su nombre lo indica, son los descendientes de la unión de europeos y amerindios. En este grupo se encuentran James Bird, Peter Erasmus, James McKay, Charles Nolin y Jerry Potts, entre otros. Estos intérpretes eran bilingües o trilinguies desde la infancia y vivían entre dos mundos. Algunos, como James McKay y Charles Nolin, llegaron a ocupar cargos en el gobierno. James McKay participó en las negociaciones de los tratados 1 a 4 como intérprete y en los tratados 5 y 6 como comisionado del gobierno (Morris 1880). Charles Nolin llegó a ser ministro de agricultura de Manitoba por algún tiempo y también fue uno de los principales instigadores de la rebelión de los Métis en 1885 junto a Louis Riel, aunque luego traicionó esta causa (Adams 1975). Por su parte, Jerry Potts trabajaba para la policía montada como guía (St. Germain 2001)

- Los misioneros: Varios misioneros, tanto católicos como protestantes, participaron en las negociaciones como intérpretes. Estos misioneros se habían instalado en territorio amerindio y conocían las costumbres y las lenguas indígenas, aunque probablemente no tan bien como los Métis. En este grupo destacan el Reverendo Henry Cochrane y los Reverendos Georges McDougall y John McDougall, padre e hijo. John McDougall es autor de tres libros sobre la vida en las praderas. Entre los católicos, podemos mencionar a Jean L'Heureux y a Constantine Scollen.

\subsection{El contexto de las negociaciones}

En los últimos treinta años, el tema de los tratados sobre cesión de territorio firmados con los amerindios canadienses ha sido objeto de un gran número de estudios, que se basan tanto en documentos de archivos como en entrevistas con amerindios ancianos que aún conservan la tradición oral de los protagonistas de los hechos.

De estos estudios se desprenden dos elementos fundamentales sobre el contexto histórico de las negociaciones.

En primer lugar, existían grandes diferencias culturales entre las dos partes. A pesar de haber tenido contacto con los colonizadores por dos siglos, los indios aún conservaban su lengua y su modo de vida. Vivían de la caza y la pesca, y eran nómadas o semi-nómadas. No utilizaban la escritura y se basaban en la tradición oral. Lo que se decía en las discusiones era su única referencia. Para el gobierno, en cambio, el tratado escrito y firmado era la ley. Varios expertos (Fumoleau 1973, Miller 1989) han señalado que los amerindios no estaban familiarizados con conceptos como cesión, renuncia y venta de territorio. Para ellos, la tierra era un regalo espiritual de la naturaleza que compartían con todos los hombres.

En segundo lugar, las dos partes no tenían las mismas motivaciones. Los intereses del gobierno estaban claros: obtener tierras fértiles para los nuevos inmigrantes, ofrecer seguridad a los nuevos pobladores y "civilizar" a los amerindios y convertirlos en agricultores, es decir, asimilarlos al resto de la sociedad. Según Sprague (1991) y Fumoleau (1973), la firma de los tratados coincide 
con el primer auge económico de la región correspondiente, como la fiebre del oro, la expansión del ferrocarril y el descubrimiento de yacimientos petroleros o mineros. Cuando el gobierno anticipaba que las tierras serían rentables, firmaba un tratado con los amerindios para comprarlas oficialmente y evitar todo tipo de reivindicación en el futuro.

Las motivaciones del gobierno no eran sólo económicas. Como explican Stanley (1983) y Tobias (1983), a mediados del siglo XIX, las corrientes del humanitarismo y del darwinismo social gozaban de gran popularidad en Inglaterra. Este pensamiento se hizo sentir en Canadá en el marco de las relaciones con los amerindios. Éstos eran vistos como "niños" a quienes había que salvar de la extinción y la precariedad. Era necesario educarlos para que se integraran a la sociedad blanca.

Para los amerindios, los tratados debían establecer las reglas de su futura convivencia con el gobierno. En dichos tratados cedían las tierras a cambio de protección y de reconocimiento. En la segunda mitad del siglo XIX, los amerindios sufrían las consecuencias de la desaparición del búfalo, así como los estragos del alcohol y las enfermedades. Temían que su civilización se extinguiera como consecuencia del avance inevitable de la colonización y anhelaban que el gobierno los protegiera de los especuladores y les permitiera seguir su modo de vida (Miller 1989).

Aunque cabe pensar que los amerindios no fueron totalmente pasivos durante las negociaciones y obtuvieron ciertas concesiones del gobierno, como señalan Tobias (1983) y Friesen (1986), es evidente que el poder de negociación del gobierno era superior al de los amerindios, porque el primero representaba la ley y controlaba los espacios militares, legales, económicos y educativos del país. Con tratado o sin él, los colonizadores terminarían ocupando el territorio.

\subsection{El papel de los intérpretes}

Con base en los datos biográficos disponibles y las fuentes consultadas, se han formulado dos hipótesis de trabajo sobre el papel del intérprete.

1. Los intérpretes desempeñaron un papel amplio en las negociaciones en el que se superponían varios papeles al mismo tiempo. Además de mediadores lingüísticos y culturales, asumieron las funciones de negociadores, asesores culturales e informantes.

Como se dijo anteriormente, los intérpretes eran ante todo comerciantes, guías o misioneros. En esas actividades, mediaban "entre dos mundos", para retomar la frase de Kartunnen (1994), es decir, no existía una frontera definida entre los papeles de "intérprete" "asesor cultural", "negociador" e "informante". Es posible que estos papeles fueran incompatibles entre sí y que esa ambigüiedad afectara la neutralidad del intérprete. Este conflicto de papeles en el intérprete ya ha sido planteado por Anderson (1976):

The interpreter commonly serves two clients at the same time. He is the "man in the middle" with some obligations to both clients, and these obligations may not be entirely compatible. (Anderson 1976: 216).

Por otro lado, el análisis de algunos testimonios permite pensar que el gobierno seleccionaba a los intérpretes por la influencia que éstos ejercían en los amerindios. En otras palabras, su misión principal era convencerlos de firmar el tratado. En su obra sobre los tratados 1 a 7 , el entonces gobernador de Manitoba y los territorios del noroeste, Alexander Morris, describe a James McKay, funcionario gubernamental e intérprete, de la siguiente manera:

(...) a half-breed intimately acquainted with the Indian tribes and possessed of much influence over them.

(Morris 1880: 25).

Los intérpretes ocupaban un lugar importante en las negociaciones; prueba de ello es que su firma aparece en todos los tratados. Su palabra vale tanto como la de los agentes gubernamentales, los indios y los distintos testigos. En los testimonios de los comisionados, puede leerse que muchas veces el intérprete iba solo a negociar con los indios en nombre del gobierno. En un informe oficial fechado el 14 de octubre de 1873, el gobernador Morris relata que, al tercer día, las negociaciones estaban en un punto muerto, por lo que decide enviar a los intérpretes a "aconsejar" a los indios: 
[...] I requested the Hon. James McKay, Charles Nolin and Pierre Levailler to go down to the Indian Council and, as men of their own blood, give them friendly advice. (Morris 1880: 49).

De esta primera hipótesis deriva una segunda hipótesis:

2. Los intérpretes ocupaban una posición de poder. Con dicho poder, tuvieron tres opciones: a. Ser neutrales y facilitar el entendimiento entre las dos partes (el papel tradicional de un intérprete según las reglas actuales de la profesión).

Varios autores han tratado de caracterizar este papel con las metáforas de "eco fiel" (faithful echo), "no partidario" ("non partisan"), "conducto" ("conduit") (Pöchhacker 2004). Ahora bien, como han señalado Anderson (1976), Roberts (1995) y Wadensjö (1998), en situaciones con grandes diferencias culturales como la interpretación comunitaria y en el caso de los tratados con los amerindios, este intérprete "no partidario" no necesariamente es "invisible". Por querer facilitar el entendimiento entre las dos partes, puede llegar a manipular ciertos elementos comunicativos, omitiendo y modulando enunciados que resultan inadecuados o insultantes para la otra cultura. En su trabajo sobre los intérpretes con los Iroquois, Hagedorn (1988) expone que los intérpretes intervenían y adaptaban los discursos cuando lo consideraban necesario:

By subtly altering the tone or style of a speaker's remarks, the interpreter could sometimes direct the course of a series of exchanges toward a desired end or away from misunderstandings. (Hagedorn 1988: 65).

Por lo tanto, en este primer caso, aunque no toma posición a favor de alguna de las dos partes, el intérprete interviene de manera activa en el diálogo. En las siguientes dos posibilidades, el intérprete toma posición abiertamente y utiliza todos los medios de los que dispone para alcanzar sus fines:

b. Ser un instrumento de poder y contribuir a la asimilación de los indígenas, bien sea porque tenía los mismos intereses del gobierno o por otras causas, como la evangelización. Como se dijo anteriormente, varios de los intérpretes que participaron en las negociaciones eran misioneros.

c. Ser un instrumento de defensa de las minorías y lograr hacer escuchar su voz.

En esta etapa preliminar de la investigación, no se dispone aún de suficientes datos para saber si alguna de estas tres posiciones predominó en los tratados estudiados. De cualquier forma, por lo particular del contexto en que trabajaban los intérpretes, se cree que pocas veces fueron completamente neutrales.

\section{Conclusión}

Este estudio se propone llenar un vacío en la historia de la interpretación en Canadá y, como se dijo anteriormente, aportar nuevos elementos para reflexionar sobre el papel del intérprete y la dimensión sociocultural de la interpretación. Tal reflexión no sólo es útil desde el punto de vista teórico, sino que también resulta pertinente en la práctica, sobre todo en el ámbito de la interpretación comunitaria. De hecho, uno de los temas sobre los que más se debate en interpretación comunitaria es la función del intérprete. ¿Debe intentar convencer al inmigrante de seguir las instrucciones del policía o del médico, aunque contradigan creencias religiosas o costumbres culturales? Los misioneros que actuaron como intérpretes en las negociaciones de los tratados pensaban que la política de asimilación del gobierno beneficiaría a los amerindios. La presente investigación puede ayudar a entender mejor los límites y el alcance de la interpretación en contextos en los que las diferencias de estatus, de cultura y de poder afectan la actuación del intérprete y ponen en tela de juicio la imagen y la concepción que tenemos de nuestra profesión.

\section{REFERENCIAS BIBLIOGRÁFICAS}

Adams, H. (1975): Prison of Grass: Canada from the Native Point of View, Toronto, General Publishing. AlOnSO ARAgUÁs, I. (2003): "Ficción y representación en el discurso colonial: el papel del intérprete en el 'Nuevo Mundo"”, in MuÑoz MARTín, R. (ed): Congreso Nacional de la Asociación Ibérica de Traducción e 
Interpretación, vol. 1, Granada, AIETI, p. 407-419.

ANDERSON, R. B. W. (1976): "Perspectives on the Role of the Interpreter", in BRISLIN, R. W. (ed): Translation: Applications and Research, New York, Gardner Press, p. 208-228.

BAIGORRI JALÓN, J. (2003): "La investigación en historia de la interpretación de conferencias", in ORTEGA ARJONILla, E. (ed): Panorama actual de la investigación en traducción e interpretación, vol. 1, Granada, Comares, p. 341-356.

BAIGORRI JALÓN, J. (2004a): De Paris à Nuremberg : Naissance de l'interprétation de conférence, Ottawa, Presses de l'Université d'Ottawa.

BAIGORRI JALÓN, J. (2004b): Interpreters at the United Nations: A History, Salamanca, Ediciones Universidad de Salamanca.

CÁCERES WÜRSIG, I. (2004): "Breve Historia de la Secretaría de Interpretación de Lenguas", Meta 49-3, p. 609-628.

CAstilho Pais, C. (2003): "Aspectos de la traducción oral en Portugal en el siglo XVI", in SABio Pinilla, J. A. y M. D. VALENCIA (eds): Seis estudios sobre la traducción en los siglos XVI y XVII, Granada, Comares, p. 169-198. Cronin, M. (2002): "The Empire Talks Back: Orality, Heteronomy and the Cultural Turn in Interpreting Studies", in PÖchHACKer, F. and M. Shlesinger (eds): The Interpreting Studies Reader, London, Routledge.

DELISLE, J. (2003): "L'histoire de la traduction : son importance en traduction, son enseignement au moyen d'un didacticiel multimédia et multilingue", Forum 1-2, p. 1-16.

Delisle, J. et J. WOODSwORTH (eds) (1995): Les Traducteurs dans l'histoire, Ottawa, Presses de l'Université d'Ottawa.

DEMERS, G. (2003): "L'interprétation en Colombie-Britannique à l'époque des explorations par voie terrestre et de la traite des fourrures dans les comptoirs (1973-1846)", TTR XVI-2, p. 15-44.

ERASMus, P. (1999): Buffalo Days and Nights, Calgary, Fifth House Publishers, 2 ed.

FRIESEN, J. (1986): “'Magnificent Gifts': The Treaties of Canada with the Indians of the Northwest 1869-1876”, Mémoires de la Société royale du Canada 1-5, p. 41-51.

Fumoleau, R. (1973): As Long as This Land Shall Last: A History of Treaty 8 and Treaty 11 1870-1939, Toronto, MClelland and Stewart.

GaIBA, F. (1998): The Origins of Simultaneous Interpretation: The Nuremberg Trials, Ottawa, Presses de l'Université d'Ottawa.

GRAMMOND, S. (1995): Les traités entre l'État canadien et les peuples autochtones, Cowansville, Éditions Y Blais.

HAGEDORN, N. (1988): "A friend to go between them: the interpreter a cultural broker during Anglo-Iroquois Councils 1740-70", Ethnohistory 35, p. 60-80.

KARTUNNEN, F. (1994): Between worlds: interpreters, guides and survivors, New Brunswick, Rutgers University Press.

MiLleR, J. R. (1989): Skyscrapers Hide the Heavens: A History of Indian White Relations in Canada, Toronto, University of Toronto Press.

MORRIS, A. (1880): The Treaties of Canada with the Indians of Manitoba and the North West Territories, Including the Negotiations on Which They Were Based and Other Information Related Thereto, Toronto, Belford, Clark \& Co. Publishers.

PÖCHHACKER, F. (2004): Introducing Interpreting Studies, London, Routledge.

PYM, A. (1998): Method in Translation History, Manchester, St. Jerome.

ROBERTS, R. (1995): "Community Interpreting Today and Tomorrow", in CARR, S. and al. (eds): The critical link: interpreters in the community. Papers from the First International Conference on Interpreting in Legal, Health, and Social Service Settings, Amsterdam, John Benjamins, p. 7-26.

SPRAGUE, D. (1991): Canada's treaties with aboriginal people, Winnipeg, University of Manitoba.

St. Germain, J. (2001): Indian Treaty Making Policy in the United States and Canada 1867-1877, Toronto, University of Toronto Press.

Stanley, G. (1983): “As Long as the Sun Shines and Water Flows: A Historical Comment”, in GetTy, I. et A. LUSSIER (eds): As Long as the Sun Shines and Water Flows: A Reader in Canadian Native Studies, Vancouver, University of British Columbia Press, p. 1-26.

TOBIAS, J. L. (1983): "Canada's subjugation of the Plain Crees 1879-1885”, Canadian Historical Review 64, p. 519-548.

WADENSJÖ, C. (1998): Interpreting as Interaction, London, Longman. 